précédé d'un rapport particulièrement intéressant de $M$. le conseiller cotelle, et M. Thaller en a fait une annotation des Hus remarquables.

inversement, il est bien certain que si la demande en nullité de la Sociélć ćtait faile avant la déclaralion de faillite, celle-ci n'aurait plus raison d'être, et serait mème juridiqueinent impossible. I. críancier personnel de l'dssocić qui aurait obtenu la mullité de la Sociélé, et par conséquent, la confusion, le droit de faire rentrer furtivement l'apport fait pas l'associé dans le patrimoine de celui-ci, pourrait s'opposer à ce qu'une faillite, faisant obslarle à sis droits, vînt consolider sous forme d'une masse te patrimoine social, alors que ju tement, il a préalablement fait celle confusion les deux pritimoines. Les mêmes principes éclairent d'un jour whe vil les deux faces de la question.

Paul Borgacte, Avocat à la Cour d'Appel de Lyon.

\section{DISTRIBUTIONS D'ÉNERGIE}

\section{ÉLAGAgr Dis ARrRES}

Monsicur le Préfet du département de....

L'article 35 de l'arrêlé du 2 I mars 1908 dispose que " sur les voies publiques empruntécs par une distribution d'énerurie ćlectique, l'élngage des arbres plantés en bordure de ces vojes publiques, soit sur le sol des voies, soit sur les moprintés parliculières, doit être effectué aussi souvent que la sécurité de la distribution l'exige ". Cet anticle ajoute que, "sil en esl requis par le service du contrôle, l'entrepreneur de la distribulion est tenu de procéder à cet élagage, en se conformant aux instruclions du service de voirie,").

La qucstion de l'élagage devant sc poser dans tous les départements, il m'a paru ulile, pour assurer par une procédure uniforme l'exécution des prescriptions de l'arrêté précilé du 2 l mars igo8, d'adopier un modèle d'arrèté réglementaire, anquel vous devrez vous conformer lorsque vous aurez à prescrire l'élagage des arbres plantés en bordure des voies publiques empruntées par des canalisations électriques aćriennes, soit sur le sol de ces voies, soit sur les propriétés particulières.

Gi-joint co projet d'arrêté-type qui scra daté du $\mathrm{r}^{\mathrm{ar}}$ sepiernbro 1 gog. Il scra publié et affiché dans la forme ordinaire, et inséré dans le Recueil des Actes administratifs de rotre préfecture.

Paris, le $\mathrm{I}^{\mathrm{er}}$ septembre $\mathrm{r} 909$.

\section{Le Ministre des Travaux Publics, A. Mudakmand.}

\section{ARRETE}

Le Préfet du département d...

Vu la loi du 15 juin 1906 sur les distributions d'énergic clectrique ;

Vu l'arrêté du Ministre des travaux publics en date du $\therefore$ mars rgo8, pris en exéculion de l'article rg de ladite loi ; ct notnmment l'article 35 dudit arrêté ;

Considérant que, sur les voies publiques empruntées par une distribution d'énergie électrique, les branches des arbres plantés sur ces voies ou faisant saillie hors des propriétés riveraines peuvent nuire à la sécurité de la distribution s'il n'est pas procédé en temps utile à leur élagage.

Arrête :

Article premier. - Sur les voies publiques empruntées par les distributions d'énergie électrique dans le départe- ment d..., il sera procédé par l'entrepreneur de chaque distribution à l'élagage des arbres plantés en bordure de ces voies publiques, soit sur le sol de ces voies, soit sur les propriélés parliculières, aussi souvent que la nécessité en sera reconnue par cet entrepreneur dans l'intérêt de la sécurite de la distribution, ou toutes les fois qu'il en sera requis pax l'ingénicur en chef du contròle des distributions d'énergie électrique.

Art. 2. - Dans tous les cas, avant de commenicer les travaux, l'entrepreneur doit en donner avis, huit jours au moins à l'avance : - au service du contrôle ; - aux services de voierie intéressés ; - aux propriétaires de toutes planlations devant ĉtre touchécs par les travaux.

Art. 3. - Les intéressís sont tenus de permettre et de faciliter l'exécution des travaux.

Art. 4. - En cas d'opposition formulée par le service de voirie ou par un propriétaire dans un délai de huit jours à partir de l'avertissement prévu à l'article 2 ci-dessus, et sur la demande de l'entrepreneur, l'exéculion de l'élagage peut être ordonnée par l'ingénieur on chef du contrôle, étant entendu que, par application de l'article 57 du décret du 3 avril 1908 , l'entrepreneur de la distribution reste entièrement responsable de tous les dommages qui pourraient être causés par l'cxécution de l'élagage.

Art. 5. - En cas d'urgence, l'entrepreneur peut procéder à l'exécution immédiate des travaux, à charge d'en aviser en même temps les intéressés.

Art. 6. - Les travaux d'élagage seront exéculés par l'entrepreneur de la distribution en se conformant aux instructions des services de voirie et sans pénétrer dans les propriétés privées.

Art. 7. - Les produits de l'élagage des arbres plantés sur les propriélés particulières seront mis à la disposition des propriétaires, qui doivent les enlever dans un délai de 48 heures.

Les produits de l'élagago des arbres planlés sur les voies publiques seront mis à la disposition des services de voirie et rangés en se conformant à leurs indications.

Art. 8. - Les conditions des travaux d'ćlagage des plantations, en dehors de ceux qu'exige la sécurilé des distributions d'ćnergie électrique, continuent d'être déterminés par les arrêtés spéciaux prévus par l'article 33 de l'arrêté réglementaire du 15 janvier 1907 sur les permissions de grande voirie.

\section{HYDRAULIQUE}

\section{DUBA'CE ET SOX CTILISATION}

Parmi les rivières de France, la Durance est certainement une de celles dont l'étude offre le plus d'intérêt. Jadis totalement inutilisée, et connue seulement par ses déprédations, elle est maintenant une source de richesses inestimables pour la Provence, à laquelle elle fournit l'eau vivifiante qui a transformé en jardins magnifiques des terrains autrefcis incultes, et dont certaines parties formaient un véritable désert.

Réservoir merveilleux de houille blanche, la Durance, dont la force motrice était à peu près complètement perdue jusqu'à ces dernières années, voit s'établir sur ses rives des usines puissantes, dont les unes permettront la création sur 
place d'industries diverses, et dont les autres porteront à des distances atteignant $200 \mathrm{~km}$., el mème davantage, la lumière et la force.

Le développement progressif des irrigations a entraîné des prélèvements d'eau de plus en plus importants sur le débil de la rivic̀re, à tel point que, pendant les périodes de sécheresse, il n'est pas possible d'alimenter complètement les nombreux canaux qui en sont dérivés. Les pénuries d'eau sont allées en s'aggravant pendant ces dernières années, occasionnant des conflits entre les riverains, et parfois de véritables désastres pour l'agriculture. La conséquence de ce fâcheux état de choses fut la mise à l'étude de la question des barrages en Durance.

Jusqu'à présent, les pénuries d'eau n’ont intéressé que l'agriculture. Nous montrerons plus loin qu'elles intéresseront bientàt lindustrie dans une très large mesure. Si dès maintenant la nécessité d’augmenter le débit d'étiage de la Durance est derenue indiscutable, elle se fera sentir encore bien plus impérieusement dans quelques années, lorsque s'échelonneront sur le cours du grand torrent alpin de nombreuses usines dont le bon fonctionnement sera intimément lié à la prospérité d'industries importantes, réparties dans toute la région du Sud-Est de la France.

La question des barrages à créer dans le bassin de la Durance a donc élé mise fort justement à l'ordre du jour.

La solulion complète du problème ainsi posé, c'est-à-dire l'aménagement rationnel des eaux de la Durance et de ses affuents, pour en tirer le meilleur parti possible dans l'intérêt de l'agriculture, de l'industrie et de l'alimentation des villes, ne peut dès maintenant être envisagée dans son ensemble. Ce sera l'œuvre des générations qui se succéderont, et qui auront à satisfaire aux besoins nouveaux à mesure qu'ils se manifesteront.

Mais au moment où l'on se prépare à faire un premier pas, très important, dans cette voie féconde de l'amélioration du régime de nos rivières alpestres, par la création du barrage-réservoir de Gréoulx, sur le Verdon, il nous paraît intéressant de résumer les éludes qui ont été faites, et de décrire sommairement un deuxième projet, de plus grande envergure, dont la réalisation assurerait à l'agriculture de nouvelles ressources en eau, et remédierait aux pénuries d'eaux industrielles dont on se préoccupe dès maintenant.

Ce dernier point de vue est d'ailleurs tout à fait d'actualité, car, de novembre r go8 jusqu'en mars rgog, les débits de la Durance et de ses affluents se sont maintenus à un étiage exceptionnellement faible.

Avant d'aborder la question des barrages, nous dirons quelipues mots du régime de la Durance, de l'utilisation actuclle de ses eaux et de ce qu'elle pourra devenir plus tard.

\section{Régime de la Durance}

La Durance se forme à Briançon, à une altitude d'environ $1.200 \mathrm{~m}$., par la réunion de la Clarée, de la Guisanne et de la Cerveyrette. Elle traverse le département des HautesAlpes, puis celui des Basses-Alpes, passe entre les départements des Bouches-du-Rhône et de Vaucluse et se jette dans le Rhône, près d'Avignon, après un parcours de 293 kilomètres depuis Briancon.

Affluents. - Ses principaux affluents sont : La Gyronde, qui descend du massif du Pelvoux ; Le Guil, qui est l'émissaire de l'importante vallée du Queyras ; I'Ubaye, qui passe à Barcelonnette et dont la longueur n'est pas inférieure à $80 \mathrm{~km}$.; Le Büch, long de go $\mathrm{km}$., qui se jette dans la
Durance près de Sisteron ; La Bléone, qui passe à Digne ; L'Asse ; le Largue ; Le Verdon, qui est l'aflluent le plus important, et dont le cours a une longueur de $180 \mathrm{~km}$.; enfin le Coulon, qui pase à Apt.

Pente. - La pente de la Durance va en diminuant de l'amont vers l'aval, phénomène que l'on constate d'ailleurs sur presque tous les cours d'eau. Mais la diminution de pente ne se produit que lentement dans la Durance, tout ou moins en aval du confluent de l'Ubaye. Entre ce point ( $70 \mathrm{~km}$ ) et le conlluent du Buëch ( $135 \mathrm{~km}$ ), la pente moyenne est de $3^{\mathrm{m}} 3$ o par kilomètre, et elle est encore de $2^{\mathrm{m}}{ }_{1}^{5}$ entre le pont de Mirabeau (204 km 5) et le pont de Mallemort ( $249 \mathrm{~km})$. On peut donc dire que la Durance a une forte pente sur toute la longueur de son cours. C'est ce qui est la cause de son régime torrentiel.

Flotlage. - La Durance coule dans un lit de cailloux très large dont elle n'occupe habituellement qu'une faible partie. Son lit mineur se déplace souvent à la suite des crues. Aussi celte rivière n'est-elle pas navigable, et n'offre-t-elle an flottage que des conditions médiocres. Néanmoins, le flottage a eu une certaine importance avant la création du chernin de fer de Marseille à Briançon. Mais depuis une vingtaine d'années, ce mode de transport très primitif est à peu près abandonné. Un seul radelier, dernier représentant des temps passés, s'obstine encore à confier au cours capricieux de la Durance quatre à cinq radeaux par an. Mais lui-même semble se lasser d'un métier où les peines et les risques dépassent les bénéfices, car en 1907 , il n'a fait descendre que deux radeaux, et autant l'année dernière.

Si le flottage n'a aucune importance, la flottabilité de la Durance, par contre, en a une très grande au point dè vue légal et administratif. Les droits qui sont reconnus aux riverains des cours d'eau non navigables ni flottables par le Code civil et la loi de 1898 , n'ont pas été accordés aux riverains des rivières navigables ou flottables. Le régime légal de ces dernières est caractérisé par les deux principes suivants : $\mathbf{I}^{\circ}$ L'Etat est propriétaire du lit qui fait partie du domaine public ; $2^{\circ}$ Les riverains n'ont aucun droit sur les eaux.

Ces principes ont pour conséquence de dispenser l'industriel qui veut créer une chute de traiter avec les riverains du cours d'eau, ce qui est un grand avantage pour lui. Sur les cours d'eau non navigables ni flottables, il est nécessaire d'acquérir les droits de riveraineté, ce qui ajoute une difficulté sérieuse à celle toujours très grande que comporto l'acquisition à l'amiable des terrains nécessaires au passage du canal.

Par contre, il faut payer une redevance à l'Etat pour' l'usage de la force motrice des cours d'eau navigables ou flottables. Cette redevance, fixée au dixième de la valeur locative de la force brute de la chute, a reçu, suivant les cas, des valeurs très diverses, qui sont comprises habituellement entre un franc et trois francs par cheval et par an.

La Durance est classée comme rivière flottable à partir du pont de Saint-Clément.

Débits. - La Durance présente deux périodes de basses eaux, l'une en été, l'aulre en hiver, séparées par des périodes de crues.

En hiver, la pluie est remplacée par de la neige dans la plus grande partie du bassin. Cette neige ne fond que dans les régions basses, et n'alimente que faiblement la rivière, dont le débit est alors formé principalement par les écoulements des sources. Il en est ainsi, normalement, depuis le 
commencement de décembre jusqu'au milieu de mars. G'est. l'étiage d'hiver.

A partir du 15 mars, la température s'élève et la durée du jour augmente rapidement. La fonte de la neige se met on train, lentement d'abord, mais elle ne larde pas a s'accentuer pour atteindre son maximum d'intensité en mai et juin. La Durance est alors en crue: Si, à la fonte des neiges, viennent s'ajouter des pluies abondantes, les crues peuvent devenir exceptionnellement fortes. C'est en avril et mai qu'elles sont le plus à redouter.

Mais le slock des neiges accumulées par l'hiver n'est pas inépuisable. Peu à peu on voit les montagnes se dépouiller de leur blanche parure et prendre leur aspect d'été. C'est au mois de juin que la consommation de neige est la plus forte. Aussi, dès le commencement de juillet, la diminution du débit cut-clle sensible. Pendant l'été, les pluies sont assez lares, el il survient souvent de longues periodes de sécheresse. Il se produit alors un nouvel étiage en août et septembre. Puis viennent les pluies d'automne qui provoquent des crucs plus ou moins fortes, surtout en octobre et novembre. Mais le froid arrive peu à peu, transformant la pluie en neige, ot ramenant insensiblement l'étiage d'hiver.

Telle est, en quelques mots, l'évolution qui se produit périodiquement dans le débit de la Durance, et que nous allons préciser davantage dans ce qui va suivre.

La connaissance des débits d'une rivière telle que la Durance, pendant une période suffisamment longue, présenle un très grand intérêt pour l'agriculture, et surtout pour l'industric. On y parvient au moyen d'observations lyydrométriques faites régulièrement tous les jours à un certain nombre d'échelles, et en exécutant de temps en temps dles jaugeages.

Lorsque, vers 1895 , nous commençâmes nos études du régime de la Durance, les observations antérieures étaient assez rares, surtout en ce qui concerne les jaugeages. Nous trouvâmes néanmoins, dans les archives de notre service, les courbes des débits de la Durance pour les années 1862 à 866, qui avaient été élablies à l'occasion d'études dont nous parlerons plus loin. Depuis cette époque, personne ne s'était plus occupé du régime de la Durance, tout au moins dans le département des Hautes-Alpes. L'indifférence mar‘uée à cel égard par nos prédécesseurs peut paraître regretlable anjourd hui, mais elle s'explique, car ces études n'auraient eu à l'époque aucun objet pratique. Mais il n'en était plus de même à la fin de l'annéc 1895 , alors que venait de se poser le problème des pénuries d'eau en Durance et fue, d'autre part, la houille blanche commencait à devenir d'actualité.

Dès celle époque, les observations hydrométriques fonctionnaient régulièrement au pont de Rousset, mais on ne faisait point de jaugeages. Nous résolûmes de combler cette lacune, ct nous fîmes exécuter dans ce but, chaque année, quelques jaugeages, principalement en temps de basses caux. Chacune de ces opérations consitait à déterminer la section mouillée de la Durance par des sondages, et à prenIre quelques vitesses de surface au moyen de flotteurs. Ceite méthode, la seule qu'il nous fût possible d'employer, ne donne pas des résultats très exacts. Elle a été remplacée depuis par une méthode beaucoup plus précise, fondée sur l'emploi du moulinet de Ott, qui représente aujourd'hui le lernier perfectionnement de l'ancien moulinet de Woltmann.

Depuis quelques années, le service des observations hydrométriques et jaugeages a pris une importance considérable, et a été étendu à tous les principaux cours d'eau des Alpes. Sur l'initiative de M. Tavernier, qui était alors ingénieur en chef des Hautes-Alpes, on comprit en haut lieu l'intérêt qui s'atlache à dresser une sorte d'inventaire da nos. richesses en forces naturelles. A la suite d'un accord intervenu entre les deux ministères des Travaux Publics et de l'Agriculture, un service spécial confié à MM. les Ingénieurs en chef Tavernier et DE la Brosse, fut institué dans le but d'étudier l'ensemble des questions se rattachant au recensement ét à l'utilisation des grandes forces hydrauliques des Alpes.

Grâce aux études et observations qui ont ćté organisées pär ce nouveau service, on commence aujourd'hui à avoir des données précises sur le régime de la Dusance et de ses principaux affluents. En nous bảsant sur les résultats de ces études, et sur nos observations antérieures, nous pouvons caractériser le régime de la Durance par les données suivantes :

Etiage d'hiver. - Le débit normal d'étiage en hiver est donné par la première colonne du tableau suivant. Toutefois, lorsqu'un hiver froid et sec succède à un automne qui a lui-même été très sec, les débits de la Durance peuvent descendre notablement en dessous. Les débits les plus faibles qui ont été constatés jusqu'en rgo8 sont donnés par la seconde colonne $(x)$. Ces très faibles débits ont, d'ailleurs, un caractère exceptionnel.

\begin{tabular}{|c|c|c|c|c|}
\hline & \multicolumn{2}{|l|}{ Eliage normal } & \multicolumn{2}{|c|}{ Eliage minimum } \\
\hline A Briançon, & 6 à $\quad 7 \mathrm{~m}$ & & 4,4 & \\
\hline A La Bessée, & xo à 12 & $n$ & $7, \mathbf{1}$ & 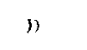 \\
\hline A Montdauphin, & 12 à 14 & $\therefore$ & 9 & ") \\
\hline A Embrun, & 20 à 23 & $\because$ & I 3,5 & $"$ \\
\hline A Rousset, & 25 à $3 o$ & $"$ & Ig & $b$ \\
\hline A Sisteron, & 35 à $/ 45$ & , & 26 & is \\
\hline A Mirabeau, & $7^{\circ}$ à $9^{\circ}$ & $\eta$ & 44 & $n$ \\
\hline
\end{tabular}

Le débit d'étiage d'hiver dure pendant quatre à cinq mois à Briançon, et présente unc très grande stabilité, à cause de la persistance du froid et de l'absence à peu près complète de pluies pendant cette période. Aussi peut-on dire qu'il n'y a pas de crues d'hiver à Briançon.

A Rousset, la période d'ćtiage est déjà sensiblement moins longue, de près' d'un mois en moyenne. Le débit est peu variable. Cependant, il peut se produire très exceptionncllement des crues de faible durée.

A Mirabeau, au contraire, l'étiage d'hiver est très instable, c'est-à-dire qu'il se produit fréquemment des crues qui peuvent même être très fortes. Cela tient à ce que le climat moyen du bassin est beaucoup plus doux que celui des Hautes-Alpes, ủe sorte que les pluies pcuvent être très abondantes, même en hiver.

Etiage d'Eté. - L'étiago d'été est caractérisé par une décroissance progressive du débit jusqu'aux pluies d'automne, avec quelquefois des relèvements accidentels dus à des pluies d'orage.

Au $x^{e r}$ août, le débit de la Durance à Rousset est habiluellement compris entre 60 et $80 \mathrm{~m}^{3}$. Il descend en septembre à 45 , et même exceptionnellement à 35 mètres cubes.

Pendant la même période, le débit à Mirabeau part de 70 à roo $\mathrm{m}^{3}$, pour descendre à 60 , et exceptionnellement à

(1) En janvier et fevrier 1949, les debits sont descendus en cerlains points légèrement on dessous des minima que nous indiqunns. In paison des circonstarices exceptionnellement difavorables qui ont amené ces débits si róduits, il semble que l'ón peut les considérer comme des maxima absolus. 
$50 \mathrm{~m}^{3}$. Il est mème descendu à $44 \mathrm{~m}^{3}$, pendant l'année $\mathrm{rgo6}$, qui restera tristement célèbre par l'extrême sécheresse, peutêtre sans précédent, de son été.

Par contre, il y a eu des années pendant lesquelles les débits ont été beaucoup plus élevés que ceux que nous venons d'indiquer. Il en est ainsi lorsqu'il se produit de fortes pluies d'orage en août et des pluies générales précoces en septembre.

Crues. - I Jes crues de printemps ou de fonte des neiges sont très régulières, quoique plus ou moins fortes. Les crues d'automne sont au contraire très variables. Certaines années, comme en 1886 et en 1907 , elles sont fortes et persistantes. Le plus souvent, elles sont d'importance moyenne et surviennent principalement en octobre et novembre. Exceptionnellement, elles peuvent ne pas se produire. Tel a été le cas des années 1899 et rgo8. Il y a alors une longue période d'étiage qui s'étend du mois d'août aú printemps de l'année suivante.

A Rousset, les crues atteignent fréquemment un débit de 500 à $600 \mathrm{~m}^{3}$. Quelquefois, elles arrivent à $1.000 \mathrm{~m}^{3}$. Le plus fort débit connu est celui de la crue du 3 I mai 1856 , qui a été évalué à 1.800 mètres cubes.

A Mirabeau, les crues sont naturellement beaucoup plus fortes, le bassin de la rivière en ce point étant plùs de trois fois plus étendu que celui en amont de Rousset. C'est surtout en aval du pont de Mirabeau que les grandes crues de la Durance sont dangereuses par les inondations et les avaries qu'elles peuvent causer. On admet que la plus forte

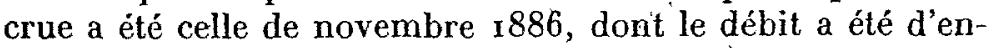
viron 6.000 mètres cubes.

Les crues de la Durance sont caractérisées par leur soudaineté. Elles se forment rapidement, et décroissent de mème. L'étale n'a qu'une durée très courte.

\section{Les Irrigations}

L'utilisation des eaux de la Durance pour l'arrosage paraît remonter au $\mathrm{i} 3^{\mathrm{e}}$ siècle, mais le premier grand canal d'irrigation, celui de Craponne, date du $16^{\circ}$. Sa création représentait à l'époque une œuvre gigantesque, et ce n'est pas sans des peines infinies que son promoteur, Adam de Craponne, put la mener à bonne fin. Parmi les anciens canaux de Provence, il convient aussi de citer ceux de Sénas et de Saint-Andiol, dans les Bouches-du-Rhône, et celui de l'Hòpital d'Avignon, dans Vaucluse. Quelques canaux furent construits au $18^{\circ}$ siècle, mais la plupart datent du $19^{\circ}$. I] en est un qui mérite une mention toute spéciale, c'est le canal de Marseille.

Vers 1840 , le développement de notre grand port méditerranéen se trouvait complètement arrêté par le manque d'eau. Des puits, et deux modestes ruisseaux, l'Huveaune et le Jarret, habituellement à sec, ne pouvaient suffire à l'alimentation d'une ville de 150.000 habitants. A tout prix, il fallait remédier à une situation qui devenait dểsastreuse pendant les périodes de sécheresse, au grand détriment de l'hygiène et de la santé publiques. Malgré des dépenses énormes et des difficultés de toute nature, la Ville de Marseille parvint à faire construire le canal, long de plus de go $\mathrm{km}$., qui devait lui amener les eaux de la Durance. Il existe sur ce canal de nombreux ouvrages d'art, dont le plus important est l'admirable pont-aqueduc de Roquefavour. Le débit dérivé est de $14 \mathrm{~m}^{3}$; il est utilisé non seulement pour l'alimentation de la ville, mais aussi pour des irrigations dans les environs. La dépense a ateint le chiffrè énorme de $4 \mathrm{I}$ millions, mais on peut dire que.cet argent a été bien employé, car la création du canal a eu pour résultat de transformer Marseille dont la population a triplé en moins de cinquante ans.

Nous donnons ci-dessous la nomenclature des principaux canaux qui sont dérivés de la Durance en aval du pont de Mirabeau, avec l'indication des débits qu'ils prélèvent normalement pendant l'été. Ces débits sont ceux qui ont été concédés, sauf pour le canal de Marseille et celui de Craponne, qui dépassent leurs dotations respectivement de $5 \mathrm{~m}^{3}$ et de 3 mètres cubes.

\begin{tabular}{|c|c|}
\hline Canal des Alpines méridionales..... & $9,5 \mathrm{~m}^{3}$ \\
\hline Canaux des Alpines septentrionales. & 12,4 \\
\hline Canal de Châteaurenard.......... & $3, \cdots$ \\
\hline Canal de Craponne ............ & $\mathbf{x} 6, \cdots$ \\
\hline Canal Crillon............. & 4,1 \\
\hline Canal de l'Hôpital d'Avignon........ & $2, n$ \\
\hline Canal de Saint-Julien............. & 4,4 \\
\hline Canal de Marseille.............. & 14," \\
\hline Canal de Carpentras............ & $10, \cdots$ \\
\hline Canal de Pertuis et Cadenet........ & l, 》 \\
\hline Canal de Peyrolles.............. & 2,3 \\
\hline Canal des Quatre-Communes....... & $2, n$ \\
\hline Autres canaux. ............. & 5,2 \\
\hline
\end{tabular}

Le total des dotations de ces canaux est de $80,5 \mathrm{~m}^{3}$, dont 53,5 pour les Bouches-du-Rhône, et 27 pour Vaucluse.

Ces canaux sont ceux qui sont dérivés directement de la Durance. Il faut y ajouter le canal du Verdon, dont la concession est de $6 \mathrm{~m}^{3}$, mais qui n'en dérive que 4,5 .

Mais ce n'est pas seulement dans ces deux départements qu'il existe des canaux d'arrosage. On peut dire que l'irrigation est utile, nécessaire même, dans tout le bassin de la Durance, c'est-à-dire aussi dans les départements des Hautes et Basses-Alpes.

Dans les Hautes-Alpes, il existe des canaux généralement peu importants, mais nombreux, dont quelques-uns sont anciens, mais dont la plupart datent du $19^{\circ}$ siècle. Ils sont dérivés principalement des affluents de la Durance, et l'on en trouve partout, même jusqu'au fond du Briançonnais où ils arrosent des terres situées jusqu'à $\mathrm{x} .500$ et $\mathrm{r} .600 \mathrm{~m}$. d'altitude. Les plus importants sont le canal Salva, à Guillestre, qui débite 600 litres par seconde; le canal de la Plaine-sousle-Roc, à Embrun (200 1.) ; le canal de Fontgillarde (40o l.); le canal des Rigues (320 l.); le canal Bouchard, à Laragne (540 1.), etc. Il convient de citer aussi le canal de Ventavon, dont la concession est de 2.500 litres et qui doit arroser la rive droite de la Durance, depuis Tallard jusqu'à Sisteron. Ce canal a été commencé en $x 885$, mais les travaux son! suspendus depuis ז 889 , par suite des dépassements qui se sont produits sur les prévisions de dépenses.

Le débit dérivé par l'ensemble des canaux d'irrigation des Hautes-Alpes peut être évalué à une dizaine de mètres cubes.

Dans les Basses-Alpes, l'arrosage se pratique à peu près de la même façon que dans les Hautes-Alpes, au moyen d'un grand nombre de petits canaux. Quelques-uns cependant ont une certaine importance : le canal de Manosque (2.0oo 1.); le canal de La Brillanne (2.00o 1.) ; le canal d'Oraison (500 1.) ; le canal des Mées (50o 1.), etc. Le débit utilisé est à peu près le même que dans les Hautes-Alpes.

Au totai, Ies canaux d'irrigation du bassin de la Durance dérivent environ 1 io $\mathrm{m}^{3}$ dont 8 ou 9 pour l'alimentation de 
Marsille of d'Mix, et le reste pour l'arrosage de terains dont la superficie esl au moins de 75.000 heclares.

Bien enlendu, l'arrosage dome des résultals d'aulant moilleurs que le climat es plus chaud. Dans les parties supérieures du bassin de la burance, les irrigations sont restreintes aux prairies et, dans une faible mesure, aux pommes de terre. $\Lambda$ mesure que l'on descend vers la Provence, les cultures irriguées augmentent comme variété et conme imporlance. Aux prairies naturelles el aux pommes de terre vicment s'ajouter les prairies arlificielles, les haricols, les légumes de loute sorle, el les arbres fruitiers.

les prairies se cultirent surtout dans les terrains maigres el peu profomls; grice au limon abondant que roule la Durance, les lerains les plus arides, quelquefois le rocher nu, se lransforment en quelques années en d'excellentes prairies. Gelles-ci donnenl on Provence quatre coupes par an. La luzerne qui nécessite, il est vrai, des terrains profonds, donne un rendemenl plus élevé, avec cinq coupes par an.

La cullure la plus produclive est celle des jrimeur fruils of légumes, qui a pris depuis une vinglaine d'années unc exlension fabuleuse dans la basse Provence, notammenl à Challeaurenard, Sainl Rémy, Carpentras, Cavaillon, elc. Gràce aux perfeclionnements des moyens de transports, les produils du sol sont expédiés à des distances de plus en plus grandes. Des fruits lòs délicals, tels que les fraiscs, arrivent en bon ćlat à Paris, en Alghlorre, en Belgique et même en Allemagne. Aussi le trafic des voies ferrées est-il augmenlé dans des proportions considerables. I a gare de Barbentane, qui dresert les principaux centres de produclion, est la gare du réscau P.-L.-M. qui, apres celle de Paris, a le plus fort tratic de orrande vilesece.

les explicalions qui précèdent, quoique sommaties, permollent de comprende naux à faible débit, ont emprunté à la rivièc une partie infime de sa puissance. Il ne pouvait d'ailleurs en être autrement dans une région purement agricole, et en raison de l'impossibilité dans laquelle on se trouvait d'utiliser et de transporter la force.

Ce fut seulement vers I $8 g_{0}$ à $189^{2}$ que les progrès réalisés

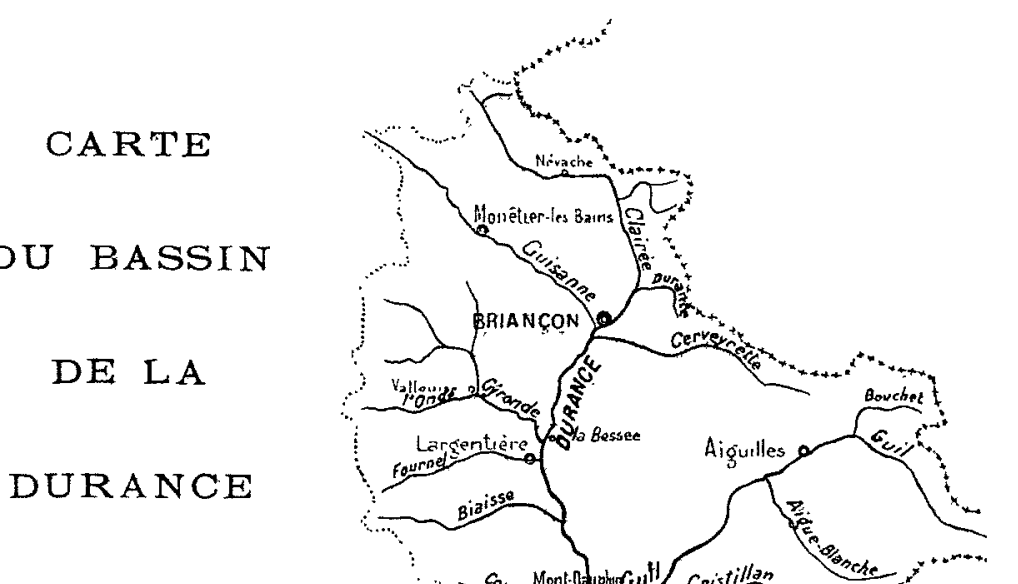

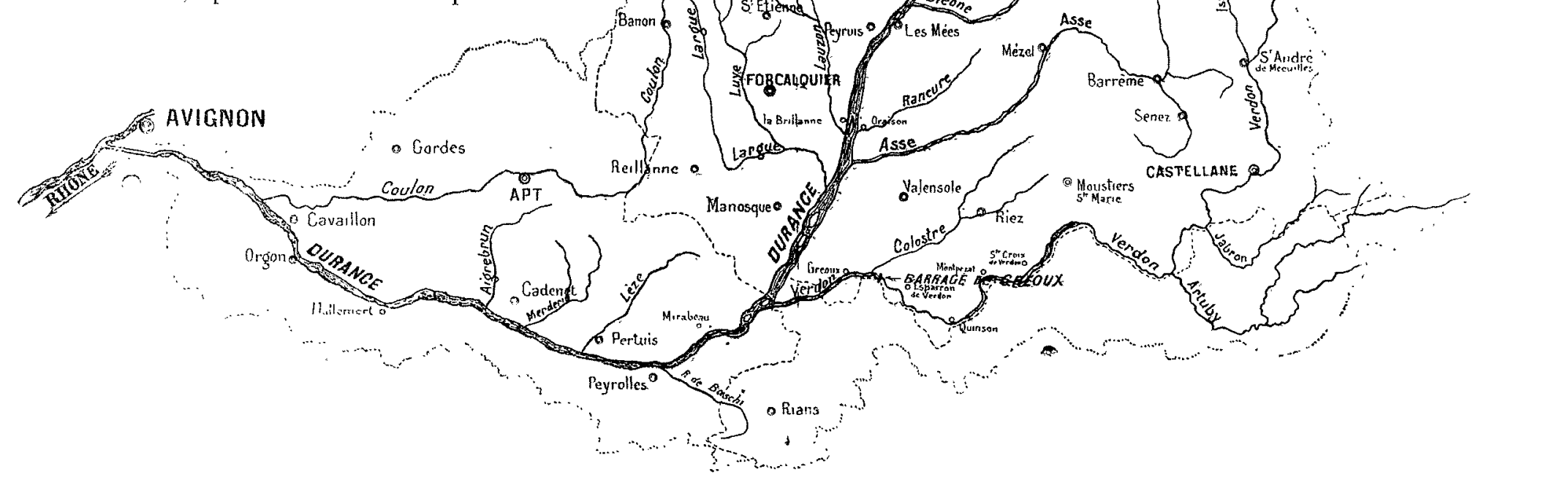

limporfance commor fule présentent les irrigations dans colle partic de la lirance, cl lintérè majeur qu'il y a à leur assurer l'eau nócessaire à leurs bosoins actucls el fiuturs.

\section{Les Usines de la Durance}

Jusqu'ì ces dernières années, l'utilisation des forces motrices de la Durance a ćté à peu près nulle. Seuls quelques moulins, dont cortains très anciens, alimentés par des ca- par l'industric électrique permirent d'cnvisager l'utilisation sux une grande échelle des forces motrices de nos cours d'eau. La nouvelle industrie de la houille blanche a pris, dès le début, un développement rapide dans l'Isère ct la Savoie, mais ce n'est que sensiblement plus tard qu'elle a fait son apparition dans le bassin de la Duranee, d'abor.d timidement, pour prendre ensuite dans ces dernières années une extension considérable. 
Si l'utilisation de la houille blanche a été relativement en retard dans le bassin de la Durance, cela tient principalement à l'éloignement des départements des Hautes et BassesAlpes de centres industriels importants. Le mouvement, parti de Grenoble, devait nécessairement mettre un temps assez long à se propager jusque dans ces deux départements, étant donné surtout que les forces naturelles du bassin de la Durance étaient encore ignorées.

De plus, au début, on avait surtout en vue des utilisations de l'énergic sur place, pour l'électrochimie et l'électrométallurgie, et l'on recherchait avant tout des forces aussi économiques que possible, et situées à proximilé des voies ferrées. Ces deux conditions se trouvaient remplies dans l'Isère et en Savoie mieux que partout ailleurs.

Enfin la Durance elle-même, avec sa pente relativement faible, qui ne dépasse pas 4 mètres par kilomètre en dessous du confluent de l'Ubaye, ne tentait pas les premiers pionniers de la houille blanche qui recherchaient surtout des cours d'eau à forte pente.

Mais si la Durance n'a qu'une pente modérée, clle a par contre un débit considérable, ce qui constitue un avantage sérieux, et de nature à compenser dans une assez largc mesure l'inconvénient signalé. Ce qui rend la Durance particulièrement intéressante au point de vue industriel, c'est sa proximité relative de Marseille et de la vallée du Rhône, qui offrent au transport de l'énergie électrique des débouchés énormes.

Etant bien placé à Gap pour suivre le mouvement industriel qui se produisait dans les régions voisines, et pour étudier les conditions d'utilisation de la Durance, nous avons signalé depuis déjà une dizaine d'années les avantages quo pourrait présenter l'aménagement des forces motrices de cetle rivière. Nous avons aujourd'hui la satisfaction de constater que nos prévisions n'étaient pas trop optimistes, et que la Durance méritait, en effet, d'attirer l'attention des industriels.

Usine de Brianģon. - La première usine qui a été établie dans le bassin de la Durance est celle de Briançon, sur la Cerveyrelte. I.a hauteur de chute est d'environ $60 \mathrm{~m}$., et le débit pouvant être dérivé de $\mathrm{x}$.ooo litres, donnant une puissance maxina de $600 \mathrm{HP}$ sur l'arbre des turbines. Cette usine produit du courant alternatif sous une tension de 2.000 volts pour l'éclairage de Briançon, celui des casernes et autres établissements militaires, et pour actionner Jes câbles porteurs qui desservent les forts de la place. I_a puis. sance utilisée est d'environ 250 chevaux. Cette usine a été construite en 1895 .

Usine de Serres. - Quelques années plus tard, un groupe d'industriels parisiens songea à créer, à Serres, sur le Buëch, une usine destinée à la fabrication du carbure de calcium. Cette usine, comportant un barrage de $6 \mathrm{~m}$. de hauteur, fut autorisée par un décret en date du 4 juillet 1899 , et les travaux furent exécutés en 1900 . La puissance maxima de l'usine est de $500 \mathrm{HP}$ pour un débit d'environ $7 \mathrm{~m}^{3}$, et une chute de $7^{\mathrm{m}} 50$. Mais ce début de l'électro-chimie dans les Hautes-Alpes ne fut pas heureux. Ie coùt de l'usine avait dépassé largement les prévisions des promoteurs de l'affaire, le débit du Buëch se révéla très insuffisant pendant une partie de l'année, et le prix du carbure de calcium tomba ǹ un chiffre dérisoire. L'usine de Serres ne put supporter la crise qui avait atteint l'industrie du carbure et elle dut cesser sa fabrication. Après liquidation, elle fut vendue à très bas prix à un industriel qui put reprendre la fabrication du carbure de calcium, grâce à un relèvement du cours de ce produit.

Usine de La Brillanne. - Les deux usines dont nous venons de parler sont en somme peu importantes. La première grande usine qui ait été établie sur la Durance est celle de Isa Brillanne. La Société de l'Energie Elcctrique du littoral Méditerranéen, qui possédait déjà un certain nombre de concessions dans les départements du Var et desi Alpes-Maritimes cherchait une force hydraulique importante pour la transporter à Marseille, d'abord pour la traction des tramways, puis pour les besoins de l'industrie et tous autres usages (I).

La difficulté n'était pas de trouver des chutes avantageuses, car sur la Durance on n'avait que l'embarras du choix ; mais il fallait résoudre le difficile problème de l'acquisition des terrains nécessaires à l'établissement du canal Ce dérivation et de l'usine. En l'absence de toute loi permettant aux industriels d'obtenir sans formalités trop longues, et sans charges excessives, la déclaration d'utilité publique, la Société de l'Energie du Littoral avait à choisir entre deux moyens: l'achat des terrains à l'amiable, ou bien la déclaration d'utilité publique par une loi spéciale, analogue à celle qui a autorisé la dérivation des eaux du Rhône près de Lyon. Le premier moyen est d'une application difficile et délicate, car la résistance d'un seul propriétaire. peut faire ćchouer l'entreprise ; de plus, on court le risque, dans certains cas, d'avoir des promesses de vente qui ne sont pas absolument régulières, ce qui peut être la source de graves difficultés ; enfin, on peut avoir à compter avec les agissements des barreurs de chutes.

Le deuxième moyen présente l'inconvénient de nécessiter une procédure longue et compliquée, et de soumettre les usagers de la force hydraulique aux conditions, parfois rigoureuses. que comporte, dans la législation actuelle, le 'égime des concessions avec déclaration d'utilité publique. Nous avons constaté maintes fois la répugnance, bien exagérée à notre avis, que manifestent les industriels à accepter ce mode de concession. Il faut d'ailleurs remarquer que, même dans ce cas, les industriels n'en jugent pas moins nécessaire d'acquérir à l'amiable la presque totalité des terrains, car les jurys d'expropriation peuvent se laisser. aller à des évaluations très exagérées lorsqu'il s'agit de țavaux pour lesquels le concours financier des départements et des communes n'est pas demandé.

L'état de choses actuel ne se prolongera d'ailleurs pas indéfiniment, et il y a tout lieu d'espérer qu'une loi sera votée prochainement qui permettra aux industriels d'obtenir la déclaration d'utilité publique dans des conditions très acceptables.

En général, on opte donc pour le premier moyen. C'est ce qu'a fait la Société de l'Energie Electrique du Littoral, qui a pu acquérir tous les terrains nécessaires pour créer une dérivation, longue d'environ $7 \mathrm{~km}$., dont l'origine est à La Brillanne (Basses-Alpes), et l'extrémité inférieure à Villeneuve, un peu en amont du confluent du Largue. L'aytorisation de dériver les eaux de la Durance ayant été accordée par un décret, les travaux furent ensuite exécutés de rgo5 à 1907 .

La prise d'cau présente cette particularité qu'elle ne comporte pas de bárrage, à cause de la grande largeur du lit de

(1) Pour la rescription des installations de cette Societé, voir $L a$ Houille Blanche d'Octobre 1907. 
la rivière, el de l'impossibilité de surélever celle-ci sans occasiomier des dommages. Les eaux de la Durance arrivent dans un avant-canal, large et profond, qui est débarrassé des graviers qu'il reçoit au moyen d'un pont roulant dragueur mû par l'électricité. Le canal, pourvu sur toùle sa longueur d'un revêtement en béton, à été construit pour un débil de $60 \mathrm{~m}^{3}$. L'tusine, que l'on voit très bien du chemin de fer, fonctionne sous une chute de $33 \mathrm{~m}$., et développe une puissance de près de 14.000 chevaux.

I a transmission de l'énergie électrique se fail sous la tension élevée de $50.00 n$ volts, qui n'avait pas encore été attcinte en Europe, tout au moins avec le courant alternatif. Une première ligue va jusqu'à Allauch, où a été élablic une puissanle station de transformation qui abạisse la tension du comrant avant son entrée dans Marseille. Une autre ligne va dans la direction d'Avignon. La distance de l'usine de La Brillanne à Marscille est d'environ roo kilomètres.

Usine de Ventavon. - Dans le courant de l'année rgoo, M. Chabrand, banquier et industriel à Gap, prenait l'initiative de faire aboutir le projet d'une usine encore plus puissanle, à établir à Ventavon (Hautes-Alpes). La question de l'acquisition des terains était particulièrement importante et difficile, car la dérivation projetée ne devait pas avoir moins de r't km. de longueur. Mais M. Chabrand finit par venis a bout de toutes les difficultés et, lorsqu'il eut obtenu toutes les promesses de vente qui lui étaient nécessaires,il demanda l'autorisation de dériver de la Durance, à La Saulce, un débit de to $\mathrm{n}^{3}$ par seconde pour actionner l'usine qu'il projetait i Ventavon. Après une instruction qui dura plusieurs années, l'autorisation demandée fut accordée par un décret en date du 29 décembre Igoh, modifić plus tard par le décret. du i 6 mars r 908 .

Pour la création de cette chute, une société anonyme a été conslituée sous le nom de Société des Forces Motrices de la Iaule-Durance. L'énergie électrique produite dans l'usine de Vontavon doil être vendue à la Société de l'Energie du Liltoral Méditerranéen pour renforcer l'alimentation de son vaste réseau primaire.

Les travaux de la dérivation et de l'usine de Ventavon ont élé commencés en rgo6. La prise d'eau comporte un barrage qui relève d'environ un mètre le niveau de la riviùre, et est pourvu de grandes vannes pour faciliter l'ćcoulement des crues, et l'entrainement des graviers. La chute nelte utilisable est de $50 \mathrm{~m}$. Avec un débil maximum de $50 \mathrm{~m}^{3}$, l'usine pourra produire une puissance de $25.000 \mathrm{HP}$ sur l'arbre des turbines. La Société de la Haute-Durance compte uliliscr des terrains plats situés vers l'extrémité du canal pour y créer un vaste bassin d'accumulation, ce qui permettra de développer un supplément de force important aux heures de la journée pendant lesquelles la consommation d'énergie est la plus forte.

L'usine comprend 6 groupes générateurs, dont 4 sont formés chacun par une turbine à axe horizonlal de 6.000 chevaux actionnant un alternateur triphasé de 4.000 kilowatts, et les deux autres par un turbine de 8.000 chevaux et un alternateur de 5.500 kilowatts. Quand l'usine sera complìtement équipée, la puissance installéc sera de flo.0oo chevaux.

A còlé de l'usine, a été construit le vaste bâtiment des transformateurs qui élèvent la tension du courant jusqu'ì 55.000 volls. Deux lignes dislinles, formées l'une par 6 cables, el l'autre par 3 cables en cuivre, amenent le courant ì La Brillanne, oì il est livré à la Société de J'Energic du I.iltoral, à une diataner dr for $\mathrm{km}$. de luaine de Ventaron.
L'énergie produite à l'usine de Ventavon sera ainsi transporléc à de grandes distances, alleignant $150 \mathrm{~km}$. à Marseille et pouvant s'élever jusqu’à r 80 el $200 \mathrm{~km}$. pour certaines autres villes.

Usine de l'Argentièrè. - Cette usine, qui est ì peu près aussi puissante que la précédente, se trouve à une quinzaine de kilomètres en aval de Briançon. Deux dérivations entièrement souterraines, longues chacune de 7 à $8 \mathrm{~km}$. amènent les caux de la Durance et de son aflluent la Gyronde à l'usine qui est établic à proximité de la gare de L'Argenlière. La hauteur de la chute est de $150 \mathrm{~m}$. Arec un débit maximum de $20 \mathrm{~m}^{3}$, la puissance disponible sur l'arbro des lurbines est de 30.000 chevaux. Elle sera utilisée en totalité sur place pour l'électro-métallurgie de l'aluminium et diverses autres industries.

Le fondateur de cette usine est M. Planche, industriel à Lyon, qui est parvenu à obtenir les droits de riveraineté el autres qui sont nécessaires pour permettre la création et le fonctionnement des deux dérivations et de l'usine. Les travaux sont cxécutés par $M$. Planche pour le comple de la Sociélé électro-métallurgique française, qui possède déjà les usines de Froges (Isère), de La Praz et de La Saussaz (Savoie).

Usine de la Roche de Rame. - Cette usine, d'une puissance maxima de 6.000 chevaux, utilise les eaux de la Biaisse, affluent de la Durance, qui se jette dans celte rivière à une vingtaine de $\mathrm{km}$. en aval de Briançon. La hauteur de chute est de $200 \mathrm{~m}$. ; le débit dérivé pourra atteindre $3 \mathrm{~m}^{3}$, mais à l'étiage ordinaire, la Biaisse ne débite guère que 600 à 700 litres par seconde, Lc promoteur de l'usine de la Biaisse est M. A. Goudet, industriel à Gap.

Cette usine est exploitée par la Société La Nitrogène pour la fabrication artificielle de l'acide nitrique ou des nitrates, par Io procédé Pauling. Celle industrie toute nouvelle, qui est basée sur la fixation de l'azote de l'air, est appelée à prendre une ćnorme extension, et à donner des débouchés pratiquement illimités à l'industrie des chutes d'eau. On sait, en effel, que l'agriculture fait une consommation énorme de nitrates, el que les nitrates artificiels, oblenus par des procédés électro-chimiques, commencent à lutter avanlageusement avec les nitrates naturels du Chili, dont l'ćpuisement d'ailleurs est inévitable d'ici peu.

Usines projetées. - Nous dirons maintenant quelques mots des usines projetées, en nous bolnant à celles qui ont fait l'objet de demandes de concession ou d'autorisalion, appuyées d'études suffisamment sérieuses, cl de promesses de. rente pour les riverainetés et les terrains nécessaires.

Sur le Guil, important affluent de rive gauche de la Durance, on projette la création d'une chute de plus de $200 \mathrm{~m}$. de hauteur, dont la puissance atteindra 90.000 chcvaux.

Une chute de $400 \mathrm{~m}$. ef d'une puissanec maxima de 4.000 chevaux, est à l'étude sur le torrent de Réallon, à Savines.

En dessous de L'Argentière, la première prise d'eau projetée sur la Durance esl au confluent du Guil. Flle doil alimenter une dérivation de $55 \mathrm{~km}$. de Iongueur, prévne pour in abib de $30 \mathrm{~m}^{3}$, aver chute de $75 \mathrm{~m}$. de hautrur a SaintAndré-d'Fmbrun. La puissance maxima est de s, sno chèvaux.

Une autre demande est relative it une prise d'ean qui serail élablie immédialement en dessous de l'usine de Saint. André, pour alimenter une dérivalion allant jusque pròs du confluent de l'Ubaye, sur laquelle semient échelommes deux 
usines, l'une à Savines, l'autre au Sauze, chacune avec $50 \mathrm{~m}$. de chule et $50 \mathrm{~m}^{3}$ de débit. I a puissance lolale maxima serait de 30.000 chevaux.

A deux kilomètres en aval du confluent de l'Ubaye, dans le défllé de Serre-Ponçon, une prise d'eau a été prévue pour l'alimentation d'une dérivation de $14 \mathrm{~km}$. de longueur, pouvańt débiter lo $\mathrm{m}^{3}$, qui alimenteràit une chute de $50 \mathrm{~m}$. de hauteur, et d'une puissance de 20.000 chevaux, à Valserres.

En sortant de l'usine de Valserres, l'eau de la Durance serait reprise dans un canal d'irrigation en partie exécuté, le canal de Ventavon, déjà cité, dont la section serait agrandie de manière à pouvoir débiter $40 \mathrm{~m}^{3}$. Une demande de concession dans ce sens est actuellement pendante, et a pour objet la création d'une usine de $30 \mathrm{~m}$. de chute el de r2.00o chevaux de puissance à La Saulce. Le canal de fuile de cette usine déboucherait à $200 \mathrm{~m}$. en amonl de la prise du canal de la Sociélć de la Hautc-Durance.

Dans la partie de la Durance comprise entre lusine de Ventavon et Sisteron, il y a un projet d'usine au Poët, avec chute d'environ $25 \mathrm{~m}$. de hauteur.

Au-delà de Sisteron, nous trouvons une demande de prise d'eau à Peipin, avec chute d'une trentaine de mètres à SaintAuban.

Près de cette dernière localité est siluée la prise d'eau du canal de Manosque. Il est question d'augmenter la section de ce canal en vue de la création d'une usine au droit de la prise du canal de La Brillanne.

Entre l'extrémité du canal de fuite de l'usine de La Brillanne et le confluent du Verdon, il y a une cinquantaine de mètres de chute à utiliser, dont la concession a étć accordée par un décret du mois d'août igos au Syndicat des Forces Motrices de la Basse-Iurance.

Enfin, deux ou trois demandeurs en concession sont en concurrence pour aménager une chute tolale de $30 \mathrm{~m}$. cntre le pont de Mirabeau et le pont de Pertuis.

Sur les affluents de la Durance, on peut encore citer une chute de $150 \mathrm{~m}$. sur l'Ubaye, et une autre de $200 \mathrm{~m}$. sur le Verdon, pour laquelle les travaux, sont commencés.

La situation actuelle de l'industric de la houille blanche dans le bassin de la Durance est résumée dans le tableau ci-joint sur lequel les usines projetées sont marqućes par une astérique.

\begin{tabular}{|c|c|c|c|c|c|}
\hline $\begin{array}{c}\text { DESTGNAITON } \\
\text { deS } \\
\text { LSIAES }\end{array}$ & $\mid \begin{array}{c}\text { COURS D'EAU } \\
\text { ulilisés }\end{array}$ & $\begin{array}{l}\text { DEBIT } \\
\text { masimum } \\
\text { prévu }\end{array}$ & $\begin{array}{c}\text { InUTEUn } \\
\text { de } \\
\text { chute }\end{array}$ & \multicolumn{2}{|c|}{$\begin{array}{l}\text { PUISSASCE } \\
\text { maxima } \\
\text { de l'usine }\end{array}$} \\
\hline 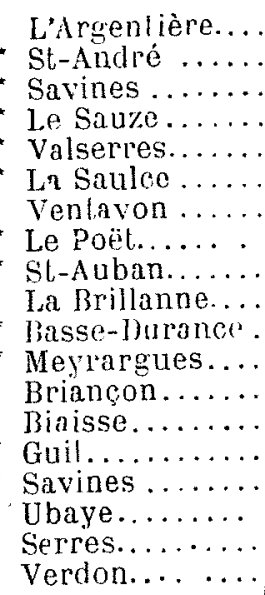 & \begin{tabular}{|c|} 
Durance \\
id. \\
jd. \\
id. \\
jd. \\
id. \\
id. \\
id. \\
id. \\
id. \\
id. \\
id. \\
Cerveyrelte \\
Biaisse \\
Guil \\
Réallon \\
Ubayo \\
Buëch \\
Verdon
\end{tabular} & $\begin{array}{l}20 \mathrm{~m}^{3} \\
30 \\
30 \\
30 \\
40 \\
40 \\
50 \\
50 \\
60 \\
60 \\
80 \\
80 \\
1 \\
3 \\
6 \\
1 \\
8 \\
7 \\
8\end{array}$ & $\begin{array}{l}150 \mathrm{~m} \\
75 \\
50 \\
50 \\
50 \\
30 \\
50 \\
95 \\
30 \\
23 \\
50 \\
30 \\
60 \\
200 \\
325 \\
400 \\
1 \div 0 \\
7 \\
200\end{array}$ & $\begin{array}{l}30.000 \mathrm{liP} \\
22.500 \\
15.000 \\
15.000 \\
20.000 \\
12.000 \\
25000 \\
12.500 \\
18.000 \\
14.000 \\
10.000 \\
2.000 \\
600 \\
6.000 \\
20.000 \\
4.000 \\
12.000 \\
507 \\
16.000\end{array}$ & 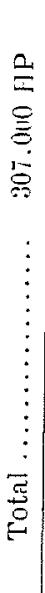 \\
\hline
\end{tabular}

Ce tableau ne comprend pas diverses usines moins importantes jui servent notamment ì l'éclairage de nombreuses communes. Il ne comprend pas non plus certaines usines projetées, mais dont la réalisation paraît plus éloignée, nolamment sur le Verdon. Nous dirons enfin que certains des chiffres de ce tableau ne sont qu'approximatifs.

On voit par l'exposé sommaire qui précède, quelle importance l'industrie des grandes chutes d'eau commence ì prendre dans le bassin de la Durance. Dès maintenant, les usines en exploitation représentent une puissance de 76.000 chevaux, et celles en construction 16.000 chevaux. Parmi celles projetées, qui alignent un total de plus de 200.000 chevaux, il y en a plusieurs qui paraissent susceptibles d'être réalisées à bref délai, et le tour des autres viendra tôt ou tard. En tenant compte des chutes utilisables industriellement, mais qui n'ont encore fait l'objet d'aucune étude sérieuse, on arrive à un total général voisin de 500.000 chevaux pour tout le bassin de la Durance.

Toules ces chutes d'eau représenteront un jour un élément important de la richesse publique. On ne saurait donc trop encourager tout ce qui pourra faciliter et développer leur utilisation.
(A suivre.)
Ingénieur en chef des Ponts et Chaussées.

\section{INSTALLATIONS HYDR0-ÉLECTRIQUES}

\section{LES INSTALLATIONS DE LA SOCIÉTÉ ANONYME DES FORCES MOTRICES DE BRUSIO}

Les installations de la Société anonyme des Forces motrices de Brusio, dont le commencement remonte au mois de juin $x_{90}$, et qui furent mises progressivement en exploitation trois ans après, exactement pendant la période qui s'étend du mois de mars r $90_{7}$ au milieu de l'année suivante, constituent le plus important transport de puissance it grande distance réalisé et fonctionnant à l'heure actuelle cn Europe, et l'un des plus importants du monde entier.

Le but poursuivi était l'alimentation en force motrice de toute la plaine Lombarde, qui s'étend au sud du lac majeur entre Milan, Novare et Lugano, région déjà siilonnée par les feeders de distribution des centrales de Vizzola, Castellanza et Turbigo appartenant à la Société italienne Lombarde " pour la distribution de l'énergie électrique ", et où l'industric cotonnière maintient, en plein activité, de grandes filatures et usines de tissage.

L'entreprise fut facilitée par l'existence à I 80 kilomètres de là, en Suisse, d'un réservoir naturel, le lac Poschiavo situé au sud du massif de la Bernina, à une altitude d'environ mille mètres, pouvant, grâce à sa situation remarquable entre deux montagnes, être relié par une canalisation de faible pente ì un point d'où il était facile d'obtenir une chutc directe de 400 mètres, ce qui permettrait, grâce à la quantitú d'eau disponible, de disposer d'une puissance considérable.

Ce lac, dont la superficie est d'environ 2 kilomètres carrés, pour une profondeur variable ne dépassant nulle part 8o mètres, reçoit les eaux que lui envoient, par l'intermédiaire de petits ruisseaux, les vallées de Campo et de Teo, ct les glaciers de Palu et de Cambrena. Il a, comme déversoir, le Poschiavino, qui sort par son extrémité sud et va se jetter dans l'Adda non loin de Tirano, après avoir franchi la frontière italo-suisse près de Campocologno, petit village de la commune de Brusio.

Depuis longtemps, on avait songé à créer dans cette région, une station hydro-électrique, mais sans approfondir le 